\title{
PD-1/PD-L1 Pathway in Breast Cancer
}

\section{Florian Schütz Stefan Stefanovic Luisa Mayer Alexandra von Au Christoph Domschke Christof Sohn}

Interdisziplinäres Brustzentrum, Universitätsfrauenklinik Heidelberg, Heidelberg, Germany

\section{Keywords}

PD1/PD-L1 - Breast cancer · Immune checkpoints . Immunotherapy

\section{Summary}

The programmed cell death-1 receptor (PD-1) is an immune checkpoint inhibitor which is expressed on the surface of immune effector cells. It is activated mainly by PD-L1 which can be expressed by all human cells. The PD-1/PD-L1 pathway plays a subtle role in maintaining peripheral T-lymphocyte tolerance and regulating inflammation. In cancer, the expression of PD-L1 seems to be one of the major immune escape mechanisms. Many studies have shown efficacy of blocking PD-1 or PD-L1 with specific antibodies like pembrolizumab or atezulizumab. In breast cancer, potential response was demonstrated in metastatic triple-negative breast cancers.

(c) 2017 S. Karger GmbH, Freiburg

\section{Introduction}

Solid tumors are initiated by a combination of mutations within their genetic information. Mutations may not only induce proliferation and invasion but also de novo antigens by changing DNA read-out. Every foreign antigen alerts the immune system, leading to an immune reaction directed against it. Evidently, in existing solid tumors, this antigen-directed immune reaction is initially ineffective or disabled or both, a phenomenon called immune escape.

\section{Immune Checkpoints}

T-cells are activated by foreign antigens presented on major histocompatibility complex and the co-expression of T-cell receptor (TCR) on the one hand, and by a concurrent co-activation of costimulatory and/or co-inhibitory signals on the other hand (fig. 1). The latter include members of the CD28/B7 family, and are known as 'immune checkpoints' $[1,2]$.

Immune checkpoints are involved in T-cell tolerance as well as activation. They play a crucial role in maintaining self-tolerance and immune homeostasis under physiological conditions, thereby protecting tissues from unnecessary damage when the immune system has efficiently cleared the pathogen [3]. Even maternal immune tolerance towards the fetus is in part regulated by checkpoint inhibitors [4].

Tumors may express immune inhibitory signals resulting in an attenuated immune reaction against the pathologic antigens [5]. Cytotoxic T-lymphocyte-associated antigen-4 (CTLA-4), the programmed cell death- 1 and its ligands (PD-1/PD-L1/2) axis, lymphocyte activation gene-3 (LAG-3), and T-cell immunoglobulin mucin-3 (TIM-3) are negative signals inhibiting T-cell immune response. In the context of tumor immunology, CTLA-4 signaling is more involved in limiting the initiation of a T-cell response in the lymph nodes, while PD-1 features more prominently later on in the process and serves to limit T-cell activity in the tumor microenvironment [6]. After TCR engagement, CTLA-4 is upregulated to attenuate T-cell responses and prevent expansion of autoreactive T-cells, primarily during the priming phase within the lymph nodes. Anti-CTLA antibodies like ipilimumab and tremelimumab were both tested in solid tumors including breast cancer with limited efficacy $[7,8]$.

\section{PD-1/PD-L1 Pathway}

PD-1 is an inhibitory immune checkpoint inhibitor which is expressed on the surface of T-cells, B-cells, natural killer T-cells,

\section{KARGER \\ Fax +497614520714

๑) 2017 S. Karger GmbH, Freiburg 


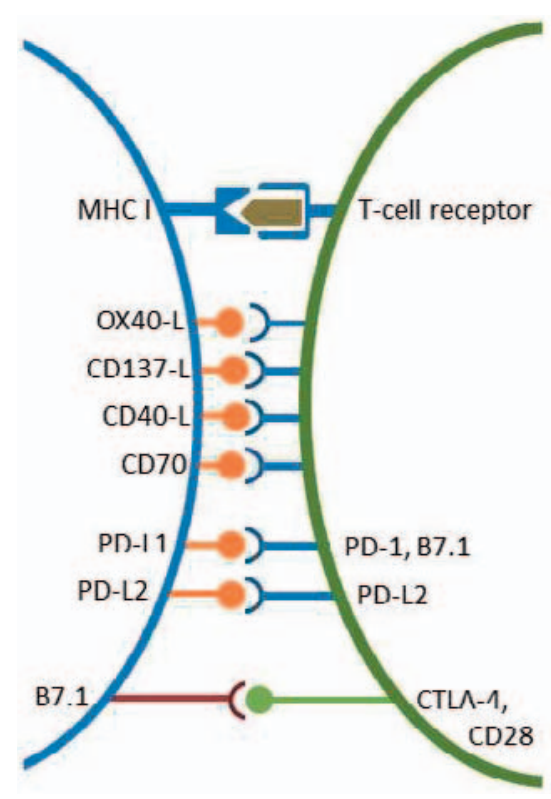

Fig. 1. Co-stimulatory and -inhibitory receptors expressed by T-cells (green) and target cells (rose). CTLA-4 or PD-1 signaling in anticancer immunotherapy [43].
Fig. 2. Blockade of

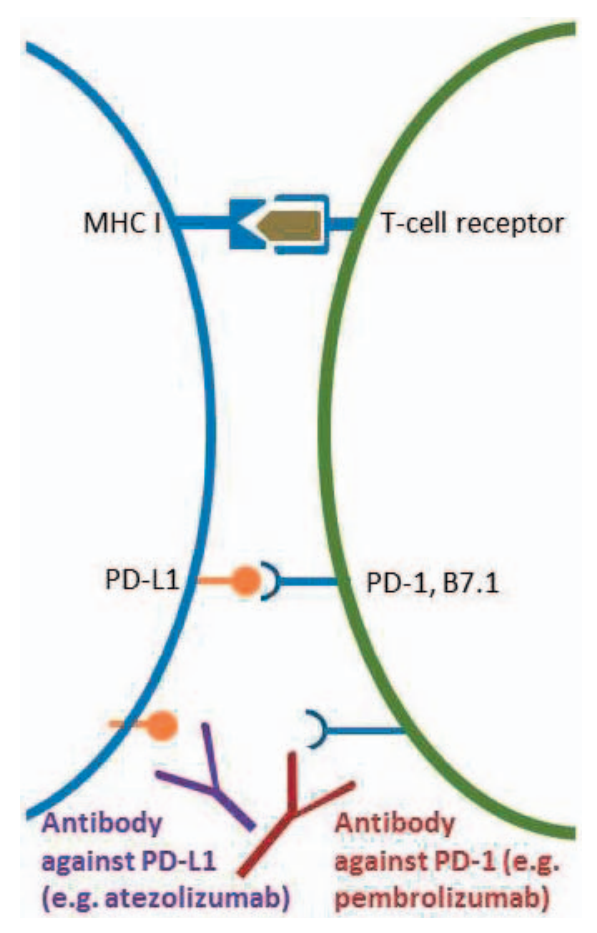

monocytes, and dendritic cells, but not resting T-cells (fig. 2). The PD-1 pathway plays a subtle role in maintaining peripheral T-lymphocyte tolerance and regulating inflammation [9].

PD-1 was originally isolated from a T-cell hybridoma undergoing T-cell receptor activation-induced cell death, hence its name, programmed cell death-1 [10]. Despite its name, PD-1 does not induce cell death directly but reduces cell growth factors as well as survival signals. PD-1 binds 2 ligands, PD-L1 (B7-H1) and PD-L2 (B7-DC) [11, 12]. Activation of PD-1 by PD-L1 or -L2 induces downregulation of T-cell activity, reduced cytokine production, T- cell lysis, and induction of tolerance to antigens [13-16]. In vitro blockade of PD-1 with monoclonal antibodies led to a 2-fold increase in cytokine production [17]. However, the in vivo activity also depends on T-cell motility as well as the duration of the interaction with antigen-presenting cells and target cells [18]. When Tcells have been activated by their TCR, PD-1 is expressed simultaneously to offer the attacked cell a way of escaping the immune reaction. PD-1 decreases once the immune response has eliminated the pathologic antigen [19]. An important role of the PD1/PD-L1 pathway has been shown in diabetes [20], cardiomyopathy [21], human immunodeficiency virus infection [22], lupus [23] and other autoimmune diseases [24], as well as in solid tumors.

Indeed, the blockade of immune checkpoints using respective monoclonal antibodies has been shown to trigger efficient antitumor responses not only in classical 'immunogenic' tumor types such as melanoma and renal cell carcinoma, but also in many other solid tumors including lung, colorectal, ovarian, esophageal, bladder, and breast cancer.

\section{PD-1/PD-L1 Pathway in Solid Tumors}

In solid tumors, the PD-1/PD-L1 inhibitory pathway can be (mis-)used to silence the immune system by increasing the expression of PD-L1 on the tumor cell surface [25]. PD-L1 expression has been associated with large tumor size, high grade, high proliferation, estrogen receptor-negative status, and HER2-positive status [26], and it is inversely correlated with survival in ovarian [27, 28] and breast cancer $[29,30]$. PD-L1 is expressed in $20 \%$ of triple-negative breast cancers (TNBCs) [31]. This indicates that although antitumor immunity is elicited against many solid tumors, it is counterbalanced by immunosuppressive factors. It was shown in vivo that PD-L1 increases tumorigenesis and invasiveness and makes tumor cells less susceptible to specific CD8+ T-cells [32]. Melanoma tumor growth is widely suppressed in PD-1 knockout mice. Furthermore, it was shown in vivo that a blockade of the PD-1/ PD-L1 pathway using specific antibodies leads to stronger tumor regression in cellular immunotherapies [33].

Translational research indicates that interferon-gamma, secreted by tumor-infiltrating cytotoxic T-cells, leads to an upregulation of PD-L1 on the surface of melanoma cells that activates the PD-1 receptor to prevent immune recognition and destruction of melanoma cells.

The goal of immune checkpoint inhibitors such as anti-CTLA-4 and anti-PD-1/anti-PD-L1 is to 'release the brakes' and enhance Tcell activation by blocking negative pathways.

\section{PD-1-Directed Treatment}

The first PD-1 antibody examined in humans was nivolumab. In 2 phase I trials with multiple tumor entities it was shown that despite the fact that only a low number of patients responded to the treatment, those few responders had an impressive long-lasting 
effect in terms of tumor remission [34]. Especially melanoma, nonsmall cell lung cancer, and kidney cancer patients showed promising results with response rates of up to $33 \%$. However, even in these early studies, immune-related adverse events grade $3 / 4$ were shown in $5 \%$ of the patients, which is now known as immune-related toxicity. These events included pneumonitis, vitiligo, colitis, hepatitis, hypophysitis, and thyroiditis, and were not correlated to dose. Even lethal events due to toxicity were reported. Hence, it is mandatory for clinicians to be able to deal with this new toxicity profile [35].

Another antibody - later named pembrolizumab - was tested in multiple phase I-III trials in solid tumors (mainly melanoma and lung cancer), showing similar results in terms of the number of responding patients, duration of response, efficacy, and toxicity compared to nivolumab.

These interesting results naturally raise the question of whether PD-1/PD-L1 pathway-directed therapy can also be of benefit in breast cancer patients. Breast cancer has several subtypes that can be analyzed by immunohistochemistry or with gene expression profiles. Especially in the estrogen-, progesterone-, and HER2-negative subtype (TNBC), in which many mutations occur that may give rise to neoantigens, we see immunogenic potential. The presence of tumor-infiltrating lymphocytes within the tumor tissue [36] as well as the prognostic value of immunity-related gene signatures in TNBC are proof of this hypothesis [37]. Especially in TNBC with its immune gene signature, PD-L1 expression can be detected in a higher proportion of tumors [38].

Single-agent pembrolizumab was tested in 27 heavily pretreated patients with metastatic PD-L1-positive TNBC within the phase Ib study, KEYNOTE-012. The antibody pembrolizumab was given intravenously at $10 \mathrm{mg} / \mathrm{kg}$ every 2 weeks. Tumor samples were screened for PD-L1 expression using a prototype immunohistochemistry assay. Patients with distinctive stromal or $\geq 1 \%$ tumor cell nest PD-L1 staining were eligible because some data suggested that patients with PD-L1 overexpressing tumors have improved clinical outcomes with anti-PD-1-directed therapy. There was a clinical benefit rate of approximately 20\%: 1 complete response (CR), 4 partial responses (PR), and 7 cases of stable disease (SD); 3 patients remained on pembrolizumab for at least 11 months. 1 treatment-related death due to disseminated intravascular coagulation was reported. Again, patients who experienced a benefit with pembrolizumab showed long duration of response (median 17.9 weeks (range 7.3-32.4 weeks)) [39]. In this small cohort, the reported side effects were comparable to those reported from melanoma and lung cancer trials. In the future, a combination with cytotoxic therapies (e.g., chemotherapy or radiation) might prove more effective than PD-1 inhibition alone. However, to date, there are only preliminary data with no efficacy data available as yet.

Atezolizumab is a human anti-PD-L1 antibody with a modified Fc region to avoid antibody-dependent cytotoxicity or complement-dependent cytotoxicity induction [40]. A total of 54 TNBC patients treated within a phase I study reached a $19 \%$ objective response rate. The duration of response ranged from 0.1 to $>41.6$ weeks.

Furthermore, the anti-PD-1 nivolumab (BMS-936558/MDX1106) and the anti-PD-L1 durvalumab (MEDI4736) are currently under investigation in breast cancer.

\section{Is PD-L1 Expression in Tumors a Predictive Biomarker?}

Since only a minority of cancer patients showed clinically relevant tumor remission after anti-PD-1 treatment, a predictive factor is required to determine patients who will derive a benefit from a checkpoint inhibitor strategy. There are several unresolved issues regarding PD-L1 analysis: variable detection antibodies, differing immunohistochemistry cut-offs, tissue preparation, processing variability, primary versus metastatic biopsies, oncogenic versus induced PD-L1 expression, and staining of tumor/stroma versus immune cells. However, despite the problems of different assays and undefined standards, it was shown in vivo that responses to antibody therapy were greater in tumors with high PD-L1 expression [41]. Trials conducted in melanoma patients reported that patients with high PD-L1 expression had a greater chance of response than those with low expression. However, there was a remarkable number of false-negative results in tumors with low PD-L1 expression even with low cut-offs; furthermore, even if high PD-L1 expression was found, $70 \%$ of melanoma tumors did not respond to anti-PD-L1 treatment.

Hence, PD-L1 expression does not seem to be a reliable predictive marker as it would exclude patients from receiving an effective treatment they may in fact respond to.

\section{Conclusion}

Immunomodulation seems to be a promising strategy in solid tumors. High immunogenicity has been described in breast cancer subtypes with a high proliferation index (TNBC, HER2). Immune checkpoints are one of the major mechanisms of immune escape. Expression of PD-L1 on tumor cells leads to lower activity of CD8+ T-cells. Antibodies against PD-1 or PD-L1 are being investigated in clinical trials. First results are promising but only a subset of patients $(20 \%)$ respond to immune checkpoint inhibitory treatment. Predictive markers are urgently needed to select those patients with the best chance for an effective treatment [42].

\section{Disclosure Statement}

The authors did not provide a disclosure statement. 


\section{References}

1 Ceeraz S, Nowak EC, Noelle RJ: B7 family checkpoin regulators in immune regulation and disease. Tends Immunol 2013;34:556-563.

2 Topalian SL, Drake CG, Pardoll DM: Immune checkpoint blockade: a common denominator approach to cancer therapy. Cancer Cell 2015;27:450-461.

3 Jung K, Choi I: Emerging co-signaling networks in T cell immune regulation. Immune Netw 2013;13:184193

4 Tripathi S, Guleria I: Role of PD1/PD-L1 pathway, and Th17 and Treg cells in maternal tolerance to the fetus. Biomed J 2015;38:25-31.

5 Pentcheva-Hoang T, Corse E, Allison JP: Negative regulators of $\mathrm{T}$-cell activation: potential targets for therapeutic intervention in cancer, autoimmune disease, and persistent infections. Immunol Rev 2009;229: 67-87.

6 Fife BT, Bluestone JA: Control of peripheral T-cell tolerance and autoimmunity via the CTLA- 4 and PD- 1 pathways. Immunol Rev 2008;224:166-182.

7 Vonderheide R, LoRusso P, Khalil M, et al.: Tremelimumab in combination with exemestane in patients with advanced breast cancer and treatment-associated modulation of inducible costimulator expression on patient T-cells. Clin Cancer Res 2010;16:3485-3494.

8 McArthur HL, Diab A, Page DB, et al.: A pilot study of preoperative single-dose ipilimumab and/or cryoablation in women with early-stage breast cancer with comprehensive immune profiling. Clin Cancer Res 2016;22:5729-5737.

9 Parry RV, Chemnitz JM, Frauwirth KA, et al.: CTLA-4 and PD-1 receptors inhibit T-cell activation by distinct mechanisms. Mol Cell Biol 2005;25:9543-9553.

10 Ishida Y, Agata Y, Shibahara K, et al.: Induced expression of PD-1, a novel member of the immunoglobulin gene superfamily, upon programmed cell death. EMBO J 1992;11:3887-3895.

11 Keir ME, Butte MJ, Freeman GJ, et al.: PD-1 and its ligands intolerance and immunity. Annu Rev Immunol 2008;26:677-704.

12 Freeman GJ, Wherry EJ, Ahmed R, et al.: Reinvigorating exhausted HIV-specific T-cells via PD-1-PD-L1 ligand blockade. J Exp Med 2006;203:2223-2227.

13 Butte MJ, Keir ME, Phamduy TB, et al.: Programmed death-1 ligand 1 interacts specifically with the B7-1 costimulatory molecule to inhibit $\mathrm{T}$ cell responses. Immunity 2007;27:111-122.

14 Freeman GJ, Long AJ, Iwai Y, et al.: Engagement of the PD-1 immunoinhibitory receptor by a novel B7 family member leads to negative regulation of lymphocyte activation. J Exp Med 2000;192:1027-1034.

15 Latchman Y, Wood CR, Chernova T, et al.: PD-L2 is a second ligand for PD-1 and inhibits T cell activation. Nat Immunol 2001;2:261-268.
16 Rodig N, Ryan T, Allen JA, et al.: Endothelial expression of PD-L1 and PD-L2 down-regulates CD8+ T cell activation and cytolysis. Eur J Immunol 2003;33:31173126.

17 Zinselmeyer BH, Heydari S, Sacristan C, et al.: PD-1 promotes immune exhaustion by inducing antiviral $\mathrm{T}$ cell motility paralysis. J Exp Med 2013;210:757-774.

18 Honda T, Egen JG, Lammermann T, et al.: Tuning of antigen sensitivity by $\mathrm{T}$ cell receptor-dependent negative feedback controls $\mathrm{T}$ cell effector function in inflamed tissues. Immunity 2014;40:235-247.

19 Vibhakar R, Juan G, Traganos F, et al.: Activationinduced expression of human programmed death-1 gene in T-lymphocytes. Exp Cell Res 1997;232:25-28.

20 Wang J, Yoshida T, Nakaki F, et al.: Establishment of NOD-Pdcd1-/-mice as an efficient animal model of type I diabetes. Proc Natl Acad Sci U S A 2005;102: 11823-11828.

21 Okazaki T, Tanaka Y, Nishio R, et al.: Autoantibodies against cardiac troponin I are responsible for dilated cardiomyopathy in PD-1-deficient mice. Nat Med 2003;9:1477-1483.

22 Day CL, Kaufmann DE, Kiepiela P, et al.: PD-1 expression on HIV-specific T cells is associated with T-cell exhaustion and disease progression. Nature 2006;443: 350-354.

23 Nishimura H, Nose M, Hiai H, et al.: Development of lupus-like autoimmune diseases by disruption of the PD-1 gene encoding an ITIM motif-carrying immunoreceptor. Immunity 1999;11:141-151.

24 Chang TT, Jabs C, Sobel RA, et al.: Studies in B7-deficient mice reveal a critical role for B7 costimulation in both induction and effector phases of experimental autoimmune encephalomyelitis. J Exp Med 1999;190: 733-740.

25 Zielinski C, Knapp S, Mascaux C, et al.: Rationale for targeting the immune system through checkpoint molecule blockade in the treatment of non-small-cell lung cancer. Ann Oncol 2013;24:1170-1179.

26 Sabatier R, Finetti P, Mamessier E, et al.: Prognostic and predictive value of PD-L1 expression in breast cancer. Oncotarget 2015;6:5449-5464.

27 Hamanishi J, Mandai M, Iwasaki M, et al.: Programmed cell death 1 ligand 1 and tumor-in filtrating CD8T lymphocytes are prognostic factors of human ovarian cancer. Proc Natl Acad Sci U S A 2007;104: 3360-3365.

28 Matsuzaki J, Gnjatic S, Mhawech-Fauceglia P, et al.: Tumor-infiltrating NY-ESO-1-specific CD8 T cells are negatively regulated by LAG-3and PD-1 in human ovarian cancer. Proc Natl Acad Sci U S A 2010;107: $7875-7880$.
29 Muenst S, Soysal SD, Gao F, et al.: The presence of programmed death 1 (PD-1)-positive tumor-infiltrating lymphocytes is associated with poor prognosis in human breast cancer. Breast Cancer Res Treat 2013; 139:667.

30 Emens L, Braiteh F, Cassier P, et al.: Inhibition of PD-L1 by MPDL3280A leads to clinical activity in patients with metastatic triple-negative breast cancer. Clin Cancer Res 2015;75(suppl 15):2860

31 Mittendorf E, Philips A, Meric-Bernstam F, et al. PD-L1 expression in triple-negative breast cancer. Cancer Immunol Res 2014;2:361-370.

32 Iwai $\mathrm{Y}$, Ishida $\mathrm{M}$, Tanaka $\mathrm{Y}$, et al.: Involvement of PD-L1 on tumor cells in the escape from host immune system and tumor immunotherapy by PD-L1 blockade. Proc Natl Acad Sci U S A 2002;99:12293-12297.

33 Kodumudi KN, Siegel J, Weber AM, et al.: Immune checkpoint blockade to improve tumor infiltrating lymphocytes for adoptive cell therapy. PLoS One 2016; 11:e0153053.

34 Topalian SL, Hodi FS, Brahmer JR, et al.: Safety, activity, and immune correlates of anti-PD-1 antibody in cancer. N Engl J Med 2012;366:2443-2454.

35 Costa R, Carneiro BA, Agulnik M, et al.: Toxicity profile of approved anti-PD-1 monoclonal antibodies in solid tumors: a systematic review and meta-analysis of randomized clinical trials. Oncotarget 2017;8:8910-8920.

36 Denkert C, Loibl S, Noske A, et al.: Tumor-associated lymphocytes as an independent predictor of response to neoadjuvant chemotherapy in breast cancer. J Clin Oncol 2010;28:105-113.

37 Cimino-Mathews A, Foote J, Emens L: Immune targeting in breast cancer. Cancer Network 2015;29:375-385.

38 Lehmann B, Bauer J, Chen X, et al.: Identification of human triple-negative breast cancer subtypes and preclinical models for selection of targeted therapies. J Clin Invest 2011;121:2750-2767.

39 Nanda R, Chow LQ, Dees EC, et al.: Pembrolizumab in patients with advanced triple-negative breast cancer: phase Ib KEYNOTE-012 study. J Clin Oncol 2016;34: 2460-2467.

40 Gibson J: Anti-PD-L1 for metastatic triple-negative breast cancer. Lancet Oncol 2015;16:e264.

41 Iwai $\mathrm{Y}$, Ishida $\mathrm{M}$, Tanaka $\mathrm{Y}$, et al.: Involvement of PD-L1 on tumor cells in the escape from host immune system and tumor immunotherapy by PD-L1 blockade. Proc Natl Acad Sci U S A 2002;99:12293-12297.

$42 \mathrm{Ma} \mathrm{W}$, Gilligan BM, Yuan J, et al.: Current status and perspectives in translational biomarker research for PD-1/PD-L1 immune checkpoint blockade therapy. J Hematol Oncol 2016;9:47.

43 Rothschild SI, Thommen S, Moersig W, et al.: Cancer immunology - development of novel anti-cancer therapies. Swiss Med Wkly 2015;145:w14066. 